\title{
Scientific journals and impact factors
}

\author{
William Hendee • Matthew A. Bernstein • \\ Deborah Levine
}

Published online: 13 December 2011

(C) ISS 2011

A research-focused scientific journal serves two main purposes. The first is to provide a forum for investigators to publish their research findings. The second is to disseminate these findings to enhance further research, and to aid others who wish to apply the findings for the benefit of society. These purposes motivate scientific journals in general and Medical Physics in particular, where the audience for the latter is principally medical physicists worldwide who are engaged in research or in the application of research results to improve patient care.

Every journal is challenged by the need to know how well it is satisfying its purposes. Measures of success include the international stature of the journal, the reputation and productivity of scientists who publish in the journal, the frequency of article downloads, the number of manuscripts submitted to the journal, and the rate at which articles are accepted or rejected. While these are useful indicators, they do not provide a single quantitative index of how well the journal is meeting its purposes.

Dr. Bernstein is Editor of Magnetic Resonance in Medicine; Dr. Levine is Senior Deputy Editor of Radiology.

\footnotetext{
W. Hendee $(\bowtie)$

Medical Physics, Medical College of Wisconsin, Milwaukee, Wisconsin, USA

e-mail: journal@aapm.org

M. A. Bernstein

Magnetic Resonance in Medicine, Mayo Clinic, Rochester, Minnesota, USA

e-mail:mrm@ismrm.org

D. Levine

Radiology, Beth Israel Deaconess Medical Center,

Boston, Massachusetts, USA

e-mail: dlevine@rsna.org
}

In 1975 the Institute for Scientific Information (now known as Thomson-Reuters or Thomson ISI) began offering journal citation reports (JCR) as part of its publication known as the science citation index [1]. The JCR's intent is to provide quantitative tools for ranking, evaluating, categorizing, and comparing journals. The major tool used by the JCR is the impact factor (IF), which is a measure of the frequency with which an "average article" in a journal is cited during a particular period. Most often the 2-year IF is used for journal ranking. As an example, the 2-year IF of a journal for 2010 is computed from data in the JCR repository as follows:

A Total number of citations during 2010 to articles published in the journal in 2008-2009

B Total number of articles published in the journal in 2008-2009

C $\quad \mathrm{A} / \mathrm{B}=2010$ impact factor

There are two principal research-focused medical physics journals. One is Medical Physics (MP) published by the American Association of Physicists in Medicine in College Park, MD, USA. The other is Physics in Medicine and Biology (PMB) published by the Institute of Physics in London, UK. In 2010, the IFs for both journals are virtually identical (MP IF 3.070, PMB IF 3.056), as they have been for most of the preceding decade. This is "good news" for the profession of medical physics, as it indicates that research is alive and well in medical physics, and that two major journals are needed to meet the publication, dissemination, and application needs of medical physicists.

The impact factor is one useful measure of a journal's importance, but it has limitations and must be used sensibly. Certainly the IF should not be interpreted as the sole criterion of worthiness, because it can be influenced by factors other than those that reflect scientific progress. For example, review 
articles are frequently cited because they summarize progress in a given area of research. A journal may be able to enhance its IF by publishing review articles, even though review articles do not directly advance the science of the discipline. Articles in a journal that address controversial issues are also often highly cited, even when they contribute little or nothing to scientific progress. Also, one or two widely-cited articles can boost a journal's IF well beyond what it might have been had the articles been published elsewhere. A few journals have tried to enhance their IFs by encouraging authors to cite articles published in the journals.

A disturbing practice gaining a foothold in some institutions in Europe and elsewhere is the use of the 2-year IF to influence faculty promotions and tenure, awarding of research grants, and other decisions that affect the careers of researchers and the research enterprise in general. That is, researchers who publish in journals with greater IFs are more highly rewarded compared with those who publish elsewhere. This practice penalizes investigators working in arcane, but potentially important, areas of research that are of less interest to widely-cited journals. It also provides inadequate recognition of visionaries and entrepreneurs who are ahead of their time or working at the margins of mainstream research, especially since the 2-year citation window is often too short for wide recognition of cutting-edge research. Other metrics that may address this shortcoming more effectively include the 5-year IF and the journal cited half-life. The latter is the median age of articles cited from a journal.

For promotion of individuals, a qualitative assessment of the importance of a scientist's work should not be supplanted by a single quantitative index such as an IF. If quantification is considered important, then the $h$ index may be a more meaningful measure than the IF for evaluating the impact of an individual scientist's work (or that of a group of individuals). The $h$ index is sometimes called the Hirsch index after the physicist who proposed it [2].
A researcher with an index of $h$ has published $h$ papers, each of which has been cited in other papers at least $h$ times [3]. Papers that have been cited fewer than $h$ times are not included in the $h$ index. The $h$ index reflects both the number of publications and the number of citations per publication. The $h$ index has its own limitations, and is useful only when comparing researchers in a single field at roughly comparable stages in their careers. Investigators working in a popular area of research are likely to garner more citations, and hence a higher $h$ index, compared with researchers working in a narrower field. Because selfcitations can raise an individual's $h$ index, it is often helpful to recalculate the $h$ index after removing them.

The impact factor plays a role in the evaluation of a journal's impact, but the role is limited and should not prevail over common sense. For the purpose of evaluating a scientist's contributions, the $h$ index may be a more meaningful measure compared with the IF.

On 20 August 2011, editors from 13 scientific journals concerned with medical imaging met in Cleveland, Ohio for the Annual Editor's Forum. Among the topics discussed was the use and misuse of journal IFs. It was decided that a small group of editors (led by William Hendee, Editor of Medical Physics) would write an editorial on the topic, with the possibility that editors may wish to revise the editorial to fit their own journals.

\section{References}

1. (http://thomsonreuters.com/products_services/science/free/essays/ impact_factor/).

2. Hirsch JE. An index to quantify an individual's scientific research output. Proc Natl Acad Sci U S A. 2005;102(46):16569-72.

3. McDonald K. Physicist proposes new way to rank scientific output. PhysOrg. http://www.physorg.com/news7971.html Accessed 8 November 2005 